\title{
MS25-01 | Structure Determination of Nano-Precipitates in Metallic Alloys using Electron Crystallography Methods
}

Meshi, Louisa (Ben Gurion University of the Negev, Beer Sheva, ISR)

Aluminides is an interesting class of materials which contain wide variety of structural complexity from simple cubes with several atoms/unit cell to quasicrystals and complex metallic alloys with hundreds atoms/unit cell. Aluminides usually appear as nanosized particles dispersed in metallic matrices. Traditional X-ray diffraction methods cannot be used for characterization of such structures due to the lack of single crystals and overlapping and/or broadening of powder diffraction peaks. Thus, Electron Crystallography (which is a combination of electron imaging and diffraction methods for solution of atomic structure of materials) is sometimes the only viable tool for this purpose due to stronger (than $\mathrm{X}$ rays) interaction with matter. The uniqueness in structure solution of complex aluminides 'structures is in lack of chemical reasonability since interatomic distances and angles vary. Thus, special methods for verification of correctness of proposed atomic model should be used. This is even more problematic, when the solution is done basing on electron diffraction (ED) data which is noisy. Atomic structures of novel complex aluminides revealed in the Th-Ni-Al and $\mathrm{Nd}$-Re-Al systems were solved using ED tomography method. Verification methods used in current research included comparison of in zone ED patterns and high resolution transmission electron micrographs to simulated (constructed based on suggested atomic model). It is interesting to note that atoms in the studied structures are situated in flat and puckered layers. This structural feature occurs in many A-T-Al aluminides (where $\mathrm{T}=$ transition metal and $\mathrm{A}=$ actinide/lanthanide), serving as additional verification of correctness of the proposed models. 